TRANSACTIONS OF THE

AMERICAN MATHEMATICAL SOCIETY

Volume 355, Number 11, Pages 4385-4396

S 0002-9947(03)03376-

Article electronically published on July 8, 2003

\title{
GROUP ACTIONS ON ONE-MANIFOLDS, II: EXTENSIONS OF HÖLDER'S THEOREM
}

\author{
BENSON FARB AND JOHN FRANKS
}

\begin{abstract}
This self-contained paper is part of a series seeking to understand groups of homeomorphisms of manifolds in analogy with the theory of Lie groups and their discrete subgroups. In this paper we consider groups which act on $\mathbf{R}$ with restrictions on the fixed point set of each element. One result is a topological characterization of affine groups in $\operatorname{Diff}^{2}(\mathbf{R})$ as those groups whose elements have at most one fixed point.
\end{abstract}

\section{INTRODUCTION}

In studying finite group actions on manifolds, one typically relates the algebraic structure of the group, the topology of the manifold, and the topology of the fixedset of the action. An example of this is Smith theory. In this paper we try to understand, in a very simple case, such relationships for actions of infinite groups. In particular we extend one of the first theorems in this direction: Hölder's Theorem.

Let $G$ be a group endowed with an order relation $<$ which is both left and right invariant. It is an old theorem of Hölder [Ho] that if $(G,<)$ is Archimedean, that is for every $g, h \in G$ there exists $n \in \mathbf{Z}$ with $g^{n}>h$, then $G$ must in fact be abelian. It was observed early on that Hölder's Theorem implies that any group of homeomorphisms of $\mathbf{R}$ which acts freely must be abelian (see, e.g., [Gh] or [FS] for a proof). This theorem may be extended to the following.

We denote by $\operatorname{Homeo}(\mathbf{R})$ the group of homeomorphisms of the real line $\mathbf{R}$, and by $\mathrm{Homeo}^{+}(\mathbf{R})$ those preserving orientation.

Theorem 1.1. Let $G<\operatorname{Homeo}(\mathbf{R})$. Suppose that there is a bounded interval $[c, d]$ so that $\operatorname{Fix}(g) \subseteq[c, d]$ for every nontrivial $g \in G$. Then $G$ is abelian.

It is not possible to weaken the hypothesis in Theorem 1.1 by allowing the interval $[c, d]$ to depend on the group element $g$ : the affine group $\Gamma$ generated by $a(x)=x+1$ and $b(x)=2 x$ has the property that each element has at most one fixed point, yet $\Gamma$ is not abelian.

Another variation is to restrict the number but not the location of fixed points.

Theorem 1.2. Let $G<\operatorname{Homeo}(\mathbf{R})$. If every nontrivial element of $G$ has precisely one fixed point, then $G$ is abelian.

In fact in this case $G$ is conjugate in $\operatorname{Homeo}(\mathbf{R})$ to a group of homeomorphisms fixing the origin and acting on each side of the origin by multiplication.

Received by the editors September 6, 2001 and, in revised form, November 29, 2001

2000 Mathematics Subject Classification. Primary 37E10.

The first author was supported in part by NSF grant DMS9704640.

The second author was supported in part by NSF grant DMS9803346. 
Characterizing affine actions. If one allows every nontrivial element of $G$ to have at most one fixed point, then a much richer collection of examples appears. For example the affine group $\Gamma=\left\langle a, b: b a b^{-1}=a^{2}\right\rangle$ mentioned above acts on $\mathbf{R}$ with every element having at most one fixed point, yet $\Gamma$ is not abelian. On the other hand this group is solvable of derived length two, i.e. its commutator subgroup is abelian.

Theorem 1.3 (T. Barbot, N. Kovacevic). Let $G<\operatorname{Homeo}^{+}(\mathbf{R})$. Suppose that every nontrivial element of $G$ has at most one fixed point. Then $G$ is metabelian and, in fact, isomorphic to a subgroup of the affine group $\operatorname{Aff}(\mathbf{R})$.

Theorem 1.3 provides an abstract isomorphism between $G$ and an affine group but does not relate the action to the standard action of $\operatorname{Aff}(\mathbf{R})$ on $\mathbf{R}$. However, using a quasi-invariant measure technique of Plante $[\mathrm{P}$ it is not difficult to prove the following.

Theorem 1.4 (N. Kovacevic). Suppose $G<\operatorname{Homeo}(\mathbf{R})$ is a nonabelian group with the property that every nontrivial element of $G$ has at most one fixed point. Then there is a continuous surjective semi-conjugacy from the action of $G$ on $\mathbf{R}$ to a subgroup of the standard action of $\operatorname{Aff}(\mathbf{R})$ on $\mathbf{R}$.

We are indebted to E. Ghys for providing us with some history of these two results. He attributes them both to V.V. Solodov, who apparently never published a proof, but did at least announce a closely related result in Theorem 3.21 of $[\mathbf{S}]$. A published proof that a nonabelian group with the property that every nontrivial element of $G$ has at most one fixed point must be metabelian was provided by T. Barbot in Theorem 2.8 of [B], at least in the case that each fixed point is a topological attractor or a repellor. Using deep results on convergence groups N. Kovacevic published proofs of both these results in $[\underline{K}$. She also considered the case that the fixed points are topologically attractors or repellors. It is likely that both Barbot and Kovacevic understood how to deal with the minor generalization to nonhyperbolic fixed points.

It is interesting to note that, prior to all of these results, J. Plante proved a result in $[\mathrm{P}$ (and described below) which easily implies Theorem 1.4 if one knows the group $G$ is solvable.

In $\$ 3.1$ we give a proof of Theorem 1.3 which takes Hölder's Theorem as a starting point and refines it by trying to reconstruct the "translation subgroup" of $G$ as the group of "infinitesimals". In this way we prove $G$ is solvable. Using the existence of a quasi-invariant measure for $G$ (established by J. Plante $[\mathrm{P}]$ ) we then conclude that the group is isomorphic to a subgroup of $\operatorname{Aff}(\mathbf{R})$.

Our main contribution to this study concerns the case when the action is $C^{2}$, in which case we are able to show that this semiconjugacy is actually a topological conjugacy. This gives a topological characterization of affine groups among all groups of $C^{2}$ diffeomorphisms of $\mathbf{R}$. E. Ghys has subsequently informed us that he is also able to prove this using techniques from Sacksteder's theory of pseudo-groups acting on the line.

Theorem 1.5 (Conjugacy). Suppose $G<\operatorname{Diff}^{2}(\mathbf{R})$ is a nonabelian group with the property that every nontrivial element of $G$ has at most one fixed point. Then there exists $h \in \operatorname{Homeo}(\mathbf{R})$ with $h G h^{-1} \in \operatorname{Aff}(\mathbf{R})$.

Theorem [1.5 is not true for abelian groups, as can be seen from an example of M. Hirsch $[\underline{\mathrm{H}}$. In this paper Hirsch gives an example of an analytic action of 
$\mathbf{Z}[1 / 2]$ on the line which is semi-conjugate to the standard one, but which has an exceptional minimal set and hence is not topologically conjugate to the standard action.

Actions on $S^{1}$. If $G$ is a group of homeomorphisms of the circle $S^{1}$, then the group $\widehat{G}$ of lifts to $\mathbf{R}$ of all elements of $G$ is given by the exact sequence

$$
1 \rightarrow \mathbf{Z} \rightarrow \widehat{G} \rightarrow G \rightarrow 1 .
$$

It follows easily from Hölder's Theorem that any group of homeomorphisms of $S^{1}$ which acts freely must be abelian. The following is a generalization of this result, allowing elements to have a fixed point.

Theorem 1.6. Let $G<$ Homeo $^{+}\left(S^{1}\right)$ be torsion-free. If every element of $G$ has at most one fixed point, then $G$ is abelian. Moreover, $G$ either acts freely or has a global fixed point.

Allowing elements of $G$ to have at most two fixed points encompasses a much richer collection of examples, for example all subgroups of $\operatorname{PSL}(2, \mathbf{R})$.

\section{ACtions by ElEments With at most one FiXed point}

In this section we consider a group $G$ acting on $\mathbf{R}$ with the property that each nontrivial element has at most one fixed point. We assume all elements preserve the orientation of $\mathbf{R}$.

\subsection{Defining the order.}

Lemma 2.1. Two elements of $G$ commute if and only if one of the following conditions is satisfied:

(1) The two elements generate a group acting freely on $\mathbf{R}$.

(2) The two elements have a common fixed point.

Proof. If the two elements, $g$ and $h$, commute, then the fixed point set of one is invariant under the other, so both have a fixed point or neither does. If both do, it is a common fixed point. If neither has a fixed point, then nothing in the group they generate has a fixed point since the element with a fixed point would also commute with $g$ and $h$.

Conversely, if $g$ and $h$ generate a group acting freely, then they commute by Hölder's Theorem. Also, if $g$ and $h$ have a common fixed point $x_{0}$, then they generate a group acting freely on the intervals $\left(-\infty, x_{0}\right)$ and $\left(x_{0}, \infty\right)$. By Hölder's Theorem we again see that they commute.

Lemma 2.2. If $g, h \in G$ and $g\left(x_{0}\right)=h\left(x_{0}\right)$ and $g\left(x_{1}\right)=h\left(x_{1}\right)$ with $x_{0} \neq x_{1}$ then $g=h$. In other words, the graphs of distinct elements of $G$ cross at most once.

Proof. The points $g\left(x_{0}\right)$ and $g\left(x_{1}\right)$ are two distinct fixed points of $h g^{-1}$. It follows that $h g^{-1}=i d$.

Definition 2.3. If $g, h \in G$ we say $g>h$ provided $g(x)>h(x)$ for all sufficiently large $x$, i.e. for all $x \in\left[x_{0}, \infty\right)$ for some $x_{0}$. We will call $g$ positive provided $g>i d$ and negative provided $i d>g$.

Since the graphs of distinct elements of $G$ cross at most once, it follows that for any distinct $g, h \in G$ either $g>h$ or $h>g$. 


\subsection{Commensurate elements.}

Definition 2.4. We say that elements $h, g \in G$ are commensurate if there are $n, m \in \mathbf{Z}$ such that $g^{-n}<h<g^{n}$ and $h^{-m}<g<h^{m}$.

Note that by taking inverses we see that $g^{-n}<h<g^{n}$ implies $g^{-n}<h^{-1}<g^{n}$. It follows that $h$ is commensurate with $g$ if and only if $h^{-1}$ is. It is an easy exercise to see that commensurability is an equivalence relation on $G$.

Lemma 2.5. Suppose $g \in G$ is positive and $h \in G$ has a fixed point. Then there exists $n \in \mathbf{Z}$ such that $h^{n}>g$ and $h^{-n}<g^{-1}$.

Proof. Replacing $h$ by $h^{-1}$ if necessary we may suppose that $h$ is positive. By conjugating $h$ we may assume that 0 is the fixed point of $h$ and that $h(x)=2 x$ for $x \geq 0$, and that for $x<0$ we either have $h(x)=2 x$ or $h(x)=x / 2$.

Suppose first that for all $x$ we have $h(x)=2 x$. We will show that for large $n, h^{n}>g$. Note that the graphs of $g$ and $h^{n}$ can intersect in at most one point by Lemma 2.2. Hence if $g(0)=0$, then the origin is the unique point of intersection of these graphs. Since $\lim _{n \rightarrow \infty} h^{n}(1)=\infty$ it follows that for $n$ sufficienly large $h^{n}(1)>g(1)$ and hence $h^{n}>g$. So we have the desired result if $g(0)=0$.

If $g(0) \geq 0$, then $g(1)>g(0)$ and since $\lim _{n \rightarrow \infty} h^{n}(1)=\infty$ we may choose $N>0$ such that $h^{n}(1)>g(1)$ for all $n>N$. Since $h(0)=0 \leq g(0)$ there is an $x_{n} \in[0,1]$ with $h^{n}\left(x_{n}\right)=g\left(x_{n}\right)$. Note that the graphs of $g$ and $h^{n}$ can intersect in at most one point by Lemma [2.2, so the fact that $h^{n}(1)>g(1)$ implies that $h^{n}(x)>g(x)$ for all $x>1$. Thus $h^{n}>g$.

Similarly if $g(0)<0$ there is an $N$ such that $h^{N}(-1)=-2^{N}<g(-1)$ and hence for any $n>N$ there is an $x_{n} \in[-1,0]$ which is the unique point of intersection of the graphs of $h^{n}$ and of $g$. Since $h^{n}(0)>g(0)$ it follows that $h^{n}(x)>g(x)$ for all $x>0$. So again $h^{n}>g$.

Consider now the case that $h(x)=x / 2$ for $x<0$. If $g(0)=0$, then the argument above remains valid, so we may assume the graph of $g$ intersects the $x$ and $y$ axes in distinct points.

Letting $n$ go to infinity, the graphs of $h^{n}$ limit on the union of positive $y$-axis and the negative $x$-axis. Also, letting $n$ go to negative infinity, these graphs limit on the union of the positive $x$-axis and the negative $y$-axis. From this it is easy to see that there exists an $n \in \mathbf{Z}$ such that the graph of $h^{n}$ intersects the graph of $g$ in two points near the two points where the graph of $g$ intersects the axes. But this is impossible by Lemma 2.2

Corollary 2.6. Let $g, h \in G$ be nontrivial. If both $g$ and $h$ have fixed points, then then they are commensurate.

Proof. Recall that $h$ and $g$ are commensurate if and only if $h^{-1}$ and $g$ are commensurate. Hence replacing $h$ and $g$ by their inverses if necessary we can assume both are positive.

By Lemma 2.5 there is an $n$ such that $h^{n}>g$. So $h^{-n}<g^{-1}$ and we have $h^{-n}<g^{-1}<g<h^{n}$. The other inequality is similar.

\subsection{Infinitesimals.}

Definition 2.7. If $g \in G$ is positive we will define the set $\mathcal{I}(g)$ of elements of $G$ which are infinitesimal with respect to $g$ by

$$
\mathcal{I}(g)=\left\{h \in G \mid g^{-1}<h^{n}<g \text { for all } n \in \mathbf{Z}\right\} .
$$


If $g$ is negative we define $\mathcal{I}(g)=\mathcal{I}\left(g^{-1}\right)$.

Lemma 2.8. If $g, g_{1} \in G$ are commensurate, then $\mathcal{I}(g)=\mathcal{I}\left(g_{1}\right)$. Also if $h \in \mathcal{I}(g)$ and $h_{1}$ is commensurate with $h$, then $h_{1} \in \mathcal{I}(g)$.

Proof. Without loss of generality we may assume both $g$ and $g_{1}$ are positive. Suppose $k>0$ and note that $g^{k}>g$ implies immediately that $\mathcal{I}(g) \subset \mathcal{I}\left(g^{k}\right)$. But also $\mathcal{I}\left(g^{k}\right) \subset \mathcal{I}(g)$ since otherwise there would be an $h \in \mathcal{I}\left(g^{k}\right)$ and an $n>0$ with either $h^{n}>g$ or $h^{n}<g^{-1}$. This would imply $h^{n k}>g^{k}$ or $h^{n k}<g^{-k}$ contradicting the fact that $h \in \mathcal{I}\left(g^{k}\right)$. We have shown that $\mathcal{I}(g)=\mathcal{I}\left(g^{k}\right)$.

Since $g$ and $g_{1}$ are commensurate there is a $k>0$ such that $g^{k}>g_{1}>g_{1}^{-1}>g^{-k}$. From this it is immediate that $\mathcal{I}\left(g_{1}\right) \subset \mathcal{I}\left(g^{k}\right)=\mathcal{I}(g)$. Since $g$ and $g_{1}$ were arbitrary positive elements we conclude that $\mathcal{I}(g)=\mathcal{I}\left(g_{1}\right)$.

To prove the second assertion of the lemma, suppose $h_{1}$ is commensurate with $h$ and $h \in \mathcal{I}(g)$. We may assume both $h$ and $h_{1}$ are positive. Then there exists $k>0$ such that $h^{-k}<h_{1}^{-1}<h_{1}<h^{k}$. It follows that $g^{-1}<h^{-n k}<h_{1}^{-n}<h_{1}^{n}<h^{n k}<g$ for any $n>0$ and hence $h_{1} \in \mathcal{I}(g)$.

We note that whenever $g$ and $g_{1}$ both have fixed points they are commensurate by Corollary 2.6] and hence $\mathcal{I}(g)=\mathcal{I}\left(g_{1}\right)$ by Lemma 2.8, so we can make the following definition.

Definition 2.9. We define $\mathcal{I}$, the infinitesimals of $G$, to be $\mathcal{I}(g)$ if $G$ contains a nontrivial element $g \in G$ which has a fixed point, and by $\mathcal{I}=G$ if $G$ acts freely.

Proposition 2.10. I is a normal abelian subgroup of $G$ which acts freely on $\mathbf{R}$.

Proof. First we note that if $G$ acts freely, then the result is immediate from Hölder's Theorem and the definitions.

Hence we may assume that $G$ contains a nontrivial positive element $g$ with a fixed point. Clearly we may assume there is a nontrivial element $h \in \mathcal{I}(g)$.

Since $h \in \mathcal{I}$ if and only if $h^{-1} \in \mathcal{I}$ we need only show $\mathcal{I}$ is closed under multiplication in order to show it is a subgroup. If $h_{1}, h_{2} \in \mathcal{I}$ we must show $h_{1} h_{2} \in \mathcal{I}$. We may, without loss of generality, assume both are positive and that $h_{2}>h_{1}$. It follows that $h_{1} h_{2}<h_{2}^{2}$ and hence that $\left(h_{1} h_{2}\right)^{-n}<i d<\left(h_{1} h_{2}\right)^{n}<h_{2}^{2 n}<g$ for all $n>0$. So for all $n$ we have $\left(h_{1} h_{2}\right)^{n}<g$ and consequently $g^{-1}<\left(h_{1} h_{2}\right)^{-n}$. So $h_{1} h_{2} \in \mathcal{I}$.

We note that Lemma 2.5] implies that any nontrivial element of $\mathcal{I}$ is fixed point free, so $\mathcal{I}$ acts freely and by Hölder's Theorem it is abelian.

The fact that $\mathcal{I}$ is normal follows from the observation that $h \in \mathcal{I}(g)$ and $a \in G$ implies $a^{-1} h a \in \mathcal{I}\left(a^{-1} g a\right)$. But as $g$ and $a^{-1} g a$ both have fixed points they are commensurate by Corollary 2.6 and hence $\mathcal{I}\left(a^{-1} g a\right)=\mathcal{I}(g)=\mathcal{I}$ by Lemma 2.8

2.4. At most one fixed point implies solvable. The following result of T. Barbot appears as Theorem 2.8 of [B]. It was apparently known to Solodov.

Theorem 2.11. Let $G<\mathrm{Homeo}^{+}(\mathbf{R})$. Suppose that every nontrivial element of $G$ has at most one fixed point. Then $G$ is solvable with derived length at most two.

Since $\mathcal{I}$ is a normal abelian subgroup it suffices to prove that $G / \mathcal{I}$ is abelian since that implies that $[G, G] \subset \mathcal{I}$. If $G$ acts freely, then $G=\mathcal{I}$ and the result is obvious, so we may assume there exists $g \in G$ which has a fixed point and that $\mathcal{I}=\mathcal{I}(g)$. 
We define an order $\succ$ on $G / \mathcal{I}$ by $a \mathcal{I} \succ b \mathcal{I}$ if and only if $a b^{-1}$ is positive and not in $\mathcal{I}$. To see this is well defined we must show that $a b^{-1}>i d$ and $a b^{-1} \notin \mathcal{I}$ implies that $\left(a h_{1}\right)\left(b h_{2}\right)^{-1}>i d$ and $\left(a h_{1}\right)\left(b h_{2}\right)^{-1} \notin \mathcal{I}$, for any $h_{1}, h_{2} \in \mathcal{I}$.

But $\left(a h_{1}\right)\left(b h_{2}\right)^{-1}=a b^{-1} h$ for some $h \in \mathcal{I}$ since $\mathcal{I}$ is normal. Clearly $a b^{-1} \notin \mathcal{I}$ implies that $a b^{-1} h \notin \mathcal{I}$. So $\left(a h_{1}\right)\left(b h_{2}\right)^{-1} \notin \mathcal{I}$.

Also the fact that $a b^{-1} \notin \mathcal{I}$ and that commensurability is an equivalence relation implies that $a b^{-1}$ is commensurate with $g$. Hence $\mathcal{I}=\mathcal{I}(g)=\mathcal{I}\left(a b^{-1}\right)$ by Corollary 2.6. Thus $a b^{-1}>h^{n}$ for all $n$ and in particular for $n=-1$. This implies $a b^{-1} h>i d$. We have shown that the order $\succ$ on $G / \mathcal{I}$ is well defined.

We also observe that this order is both left and right invariant. To see this note that if $b_{2} \mathcal{I} \succ b_{1} \mathcal{I}$, then $a b_{2} \mathcal{I} \succ a b_{1} \mathcal{I}$ since $a b_{1} b_{2}^{-1} a^{-1}$ is a conjugate of $b_{1} b_{2}^{-1}$ and hence positive and not in $\mathcal{I}$. This proves left invariance; right invariance is similar.

We next wish to show that $G / \mathcal{I}$ is Archimedian with respect to the order $\succ$. Suppose $a \mathcal{I}$ and $b \mathcal{I}$ are positive elements of $G / \mathcal{I}$, i.e. $a \mathcal{I} \succ \mathcal{I}$ and $b \mathcal{I} \succ \mathcal{I}$. Then neither $a$ nor $b$ is in $\mathcal{I}$ so they are both commensurate to $g$ and hence commensurate to each other. We conclude that there exists $k>0$ such that $a^{k}>b$. Then $a^{k} b^{-1}$ is positive and if $a^{k} b^{-1} \in \mathcal{I}$, then $a^{k+1} b^{-1}$ is positive and not in $\mathcal{I}$. We have shown that in either case $a^{k+1} \mathcal{I} \succ b \mathcal{I}$. So $G / \mathcal{I}$ is Archimedian and hence abelian by Hölder's Theorem.

2.5. Proof of Theorem 1.2. As before there exists a total order $<$ on $G$. Since every element of $G$ has a fixed point, by Lemma 2.5 we have $\mathcal{I}=\{i d\}$. Hence $G \cong G / \mathcal{I}$ is abelian by Theorem 2.11.

2.6. Proof of Theorem 1.1. As above we get a total order $<$ on $G$. Given $g, h \in G$ their graphs do not cross each other to the right of $c$. Hence to prove $g>h$ for $g, h \in G$ it is enough to prove $g\left(x_{0}\right)>h\left(x_{0}\right)$ for any single point $x_{0}>b$. By Hölder it is enough to check that, given $h, g \in G, h^{n}>g$ for some $n \in \mathbf{Z}$. By replacing $h$ with $h^{-1}$ if necessary, we may assume that $h$ is positive. But then, for any fixed $x_{0}>c$, we have $h^{n}\left(x_{0}\right) \rightarrow \infty$, so given $g\left(x_{0}\right)$ simply pick $n$ big enough that $h^{n}\left(x_{0}\right)>g\left(x_{0}\right)$, which will imply $h^{n}>g$.

2.7. Proof of Theorem 1.6. If $G$ does not act freely, then there is an $h \in G$ with a fixed point. Call this fixed point $\infty$ and conjugate so $h(x)=x+1$ on $(-\infty, \infty)$.

Let $g$ be another element of $G$. We want to show that $g(\infty)=\infty$, i.e. $\infty$ is a global fixed point. If not, consider the graph of $g$ on $\mathbf{R}=(-\infty, \infty)$. There is $x_{0} \in \mathbf{R}$ such that $g\left(x_{0}\right)=\infty$ and $y_{0} \in \mathbf{R}$ such that $g(\infty)=y_{0}$.

This means the graph of $g$ has a vertical asymptote at $x=x_{0}$ and a horizontal asymptote at $y=y_{0}$. The graph is monotonically increasing on $\left(-\infty, x_{0}\right)$, where it is entirely above the line $y=y_{0}$ and is monotonically increasing on $\left(x_{0}, \infty\right)$, where it is entirely below the line $y=y_{0}$. That is, with respect to the axes centered at $\left(x_{0}, y_{0}\right)$ the graph lies in the second and fourth quadrants.

The line $y=x-n$ is the graph of $h^{-n}$. It intersects the horizontal asymptote at $\left(y_{0}+n, y_{0}\right)$ and the vertical asymptote at $\left(x_{0}, x_{0}-n\right)$. Define $L_{n}(x)=h^{-n}(x)=$ $x-n$.

For $n$ large we claim that the graph of this line must intersect the graph of $g$ in at least two points. To see this choose a point $\left(x_{1}, y_{1}\right)$ below the graph of $g$ and with $x_{1}>x_{0}$. Then $\left(x_{1}, y_{1}\right)$ is above the graph of $y=L_{n}(x)$ for a sufficiently large $n$. 
Thus for small $\epsilon$ we have

$$
\begin{aligned}
g\left(x_{0}+\epsilon\right) & <L\left(x_{0}+\epsilon\right), \\
L\left(x_{1}\right) & <g\left(x_{1}\right), \text { and } \\
g\left(y_{0}+n\right) & <L\left(y_{0}+n\right) .
\end{aligned}
$$

Hence the graphs intersect in at least one point in $\left(x_{0}, x_{1}\right)$ and at least one point in $\left(x_{1}, y_{0}+n\right)$.

Since we have found two points where $h^{-n}(x)=g(x)$ we have found two fixed points of $h^{n} g$ and hence contradicted our hypothesis. The contradiction arose from assuming that $\infty$ was not a fixed point of $g$. Since $g$ was arbitrary we have shown that $\infty$ is a global fixed point (under the assumption that one nontrivial element has a fixed point). Since $\infty$ is a global fixed point we can consider $G$ as a free action on $\mathbf{R}=(-\infty, \infty)$. By Hölder's theorem it is abelian.

\section{QUASI-INVARIANT MEASURES}

A basic property of groups of homeomorphisms of $\mathbf{R}$ which act freely (beyond being abelian) is that they have an invariant Borel measure. This follows from the fact that the quotient space of $\mathbf{R}$ formed by identifying orbits of a single element $h$ of $G$ is homeomorphic (or diffeomorphic if $h$ is a diffeomorphism) to $S^{1}$ and the quotient group $G /\langle h\rangle$ acts on this $S^{1}$. This action has an invariant Borel probability measure which may be lifted to $\mathbf{R}$ to give an invariant Borel measure $\mu$ for $G$ which is finite on compact sets and infinite on all of $\mathbf{R}$.

The following result of J. Plante (Theorem 4.7 of $[\mathrm{P}]$ ) asserts that this can be extended to a quasi-invariant measure for certain solvable groups.

Theorem 3.1 (J. Plante). Suppose $G<\operatorname{Homeo}(\mathbf{R})$ is solvable, and its nontrivial elements have isolated fixed points. Then there exists a $G$-quasi-invariant measure $\mu$ which is finite on compact sets.

A measure is $G$ quasi-invariant if for each $g \in G$ the measure $g_{*}(\mu)$ is equal to $A(g) \mu$ for some positive real number $A(g)$. The function $A: G \rightarrow \mathbf{R}^{+}$is a homomorphism to the multiplicative group $\mathbf{R}^{+}$. The measure $\mu$ is invariant under the subgroup which is the kernel of $A$.

If one has an invariant measure $\mu$ for a subgroup of $\operatorname{Homeo}(\mathbf{R})$, it is useful to consider the translation number which was discussed by J. Plante in $[\mathrm{P}]$. In our context this is just the rotation number for circle homeomorphisms obtained by quotienting $\mathbf{R}$ by an element of $H$.

Suppose $H$ is a subgroup of $\operatorname{Homeo}(\mathbf{R})$ which preserves a Borel measure $\mu$ that is finite on compact sets. Fix a point $x \in \mathbf{R}$ and for each $f \in G$ define

$$
\tau_{\mu}(f)= \begin{cases}\mu([x, f(x))) & \text { if } x<f(x), \\ 0 & \text { if } x=f(x), \\ -\mu([f(x), x)) & \text { if } x>f(x) .\end{cases}
$$

The function $\tau_{\mu}: G \rightarrow \mathbf{R}$ is called the translation number. The following properties observed by J. Plante in $[\mathrm{P}]$ are easy to verify.

Proposition 3.2. The translation number $\tau: G \rightarrow \mathbf{R}$ is independent of the choice of $x \in \mathbf{R}$ used in its definition. It is a homomorphism from $G$ to the additive group $\mathbf{R}$. For any $f \in G$ the set $\operatorname{Fix}(f) \neq \emptyset$ if and only if $\tau_{\mu}(f)=0$. 
If $G$ is a subgroup of $\operatorname{Homeo}(\mathbf{R})$ with the property that every nontrivial element has at most one fixed point, then we can combine the homomorphisms $\tau$ and $A$ from $[\mathrm{P}]$ to obtain a homomorphisms to the affine group $\operatorname{Aff}(\mathbf{R})$.

Lemma 3.3. Suppose $G<\operatorname{Homeo}(\mathbf{R})$ is a nonabelian group with the property that every nontrivial element has at most one fixed point. If $\mu$ is a G-quasi-invariant measure which is finite on compact sets, then a nontrivial element $g$ has exactly one fixed point if and only if $A(g) \neq 1$.

Proof. If $A(g)=1$, then $\mu$ is $g$-invariant and $\tau_{\mu}(g)$ is well defined. If $\tau_{\mu}(g) \neq 0$, then $g$ has no fixed points by Proposition 3.2 . If $\tau_{\mu}(g)=0$, then for each $x \in$ $\mathbf{R}, \mu\left(\left[x, g^{n}(x)\right)\right)$ is bounded as a function of $n$. This implies that $g$ has infinitely many fixed points and hence must be the trivial element.

Suppose now that $A(g)=\alpha \neq 1$. Replacing $g$ by $g^{-1}$ if necessary, we may assume $0<\alpha<1$. We will show that the assumption that $g$ has no fixed points leads to a contradiction. Let $x \in \mathbf{R}$ and assume without loss of generality that $g(x)<x$ for all $x \in \mathbf{R}$, so $\lim _{n \rightarrow \infty} g^{n}(x)=-\infty$. Then

$$
\mu\left(\left[g^{n}(x), x\right]\right) \leq \sum_{i=0}^{n-1} \mu\left(\left[g^{i+1}(x), g^{i}(x)\right]\right) \leq \sum_{i=0}^{\infty} \alpha^{i} \mu([g(x), x])=\frac{\mu([g(x), x])}{1-\alpha}
$$

Hence for every $x, \mu((-\infty, x])$ is finite. But this is not possible, as follows. Since $G$ is nonabelian, there is an element $h$ in the kernel of $A$. The element $h$ has the property that $\lim _{n \rightarrow \infty} h^{n}(x)=-\infty$ and $h$ preserves the measure $\mu$. Thus

$$
\mu((-\infty, x])=\sum_{i=0}^{\infty} \mu\left(\left(h^{i+1}(x), h^{i}(x)\right]\right)=\lim _{n \rightarrow \infty} n \mu((h(x), x]),
$$

which implies that for every $x, \mu((-\infty, x])$ is either 0 or infinite, a contradiction.

Thus we conclude that $g$ has a fixed point. Since $A(g) \neq 1$, it is not the identity, so it has exactly one fixed point.

3.1. Proof of Theorem 1.3. We define a function $\nu: \mathbf{R}^{2} \rightarrow \mathbf{R}$ by

$$
\nu\left(x_{0}, x_{1}\right)= \begin{cases}\mu\left(\left[x_{0}, x_{1}\right)\right) & \text { if } x_{0}<x_{1}, \\ 0 & \text { if } x_{0}=x_{1}, \\ -\mu\left(\left[x_{0}, x_{1}\right)\right) & \text { if } x_{0}>x_{1} .\end{cases}
$$

Then define $\phi: G \rightarrow \operatorname{Aff}(\mathbf{R})$ by $\phi(g)(x)=a x+b$, where $a=A(g)$ and $b=\nu(0, g(0))$.

If $g_{1}, g_{2} \in G$, then

$$
\phi\left(g_{1} g_{2}\right)=A\left(g_{1}\right) A\left(g_{2}\right) x+\nu\left(0, g_{1} g_{2}(0)\right) .
$$

However,

$$
\begin{aligned}
\nu\left(0, g_{1} g_{2}(0)\right) & =\nu\left(0, g_{1}(0)\right)+\nu\left(g_{1}(0), g_{1} g_{2}(0)\right) \\
& =\nu\left(0, g_{1}(0)\right)+A\left(g_{1}\right) \nu\left(0, g_{2}(0)\right)
\end{aligned}
$$

Therefore

$$
\begin{aligned}
\phi\left(g_{1} g_{2}\right) & =A\left(g_{1}\right)\left(A\left(g_{2}\right) x+\nu\left(0, g_{2}(0)\right)+\nu\left(0, g_{1}(0)\right)\right. \\
& =\phi\left(g_{1}\right) \phi\left(g_{s}\right)
\end{aligned}
$$

To see that $\phi$ is injective, observe that if $g$ is in the kernel of $\phi$, then $A(g)=1$ and $\phi(g)(x)=x+\nu(0, g(0))=x+\tau_{\mu}(g)$, so that $\tau_{\mu}(g)=0$. Now if $G$ is abelian,

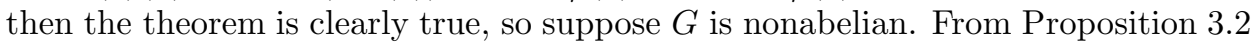


we conclude that $g$ has a fixed point and from Lemma 3.3 that it has more than one. Hence $g=i d$ and $\phi$ is injective.

3.2. Proof of Theorem 1.4 $G$ is solvable by Theorem 1.2, Hence by Theorem 3.1 there is a $G$-quasi-invariant measure $\mu$.

Define $\theta(x)=\nu(0, x)$. It was observed by J. Plante (see the remark after 4.6 of P]) that in this case $\theta$ is a semi-conjugacy, as the following calculation shows:

$$
\begin{aligned}
\theta(g(x)) & =\nu(0, g(x)) \\
& =\nu(0, g(0))+\nu(g(0), g(x)) \\
& =\nu(0, g(0))+A(g) \nu(0, x) \\
& =A(g)((\theta(x))+\nu(0, g(0)) \\
& =\phi(g)(\theta(x)) .
\end{aligned}
$$

Hence $\theta(g(x))=\phi(g)(\theta(x))$ for all $x \in \mathbf{R}$ and $g \in G$.

\section{4. $C^{2}$ ACTIONS}

In this section we prove that the semi-conjugacy in Theorem 1.4 is actually a topological conjugacy in the case that $G$ is a subgroup of $\operatorname{Diff}^{2}(\mathbf{R})$.

Definition 4.1. If $f: \mathbf{R} \rightarrow \mathbf{R}$ is a diffeomorphism and $J$ is an interval in $\mathbf{R}$, then we define the distortion of $f$ on $J$ by

$$
\operatorname{Dist}(f, J)=\sup _{x, y \in J} \log \frac{|D f(x)|}{|D f(y)|} .
$$

The following lemma is well known; a proof can be found in Chapter I, $\S 2$ of dMvS. We use the notation $|J|$ to denote the length of an interval $J$.

Lemma 4.2. If $f: \mathbf{R} \rightarrow \mathbf{R}$ is a $C^{2}$ diffeomorphism of $\mathbf{R}$ and the function $\log |D f(x)|$ is Lipschitz with Lipschitz constant $C$, then for all $n>0$,

$$
\operatorname{Dist}\left(f^{n}, J\right) \leq C \sum_{i=0}^{n-1}\left|f^{i}(J)\right| .
$$

We will also need the following lemma. This result and the proof we give are extracted from a part of the proof of a result of A. Schwartz as presented by de Melo and van Strien in Theorem 2.2 of Chapter I, $\S 2$ from $[\mathrm{dMvS}]$.

Lemma 4.3. Suppose $g: \mathbf{R} \rightarrow \mathbf{R}$ is a $C^{2}$ diffeomorphism and $\log |D g(x)|$ is Lipschitz with a uniform Lipschitz constant $C$, and suppose $J \subset \mathbf{R}$ is a closed interval with the property that

$$
\sum_{i=0}^{\infty}\left|g^{i}(J)\right| \leq 1
$$

Then there is a $\delta>0$ such that any closed interval $L$ which contains $J$ and has length $|L|<(1+\delta)|J|$ has the property that

$$
\sum_{i=0}^{\infty}\left|g^{i}(L)\right| \leq 2 .
$$


Proof. Choose $\delta$ so that $0<\delta<\min \{|J|, \exp (-2 C)\}$. We will prove by induction that $\left|g^{n}(L)\right| \leq 2\left|g^{n}(J)\right|$ for any $n \geq 0$. This is clear for $n=0$. Assume inductively that $\left|g^{k}(L)\right| \leq 2\left|g^{k}(J)\right|$ for any $k<n$.

We know by Lemma 4.2 and the induction hypothesis that

$$
\operatorname{Dist}\left(g^{n}, L\right) \leq C \sum_{i=0}^{n-1}\left|g^{i}(L)\right| \leq 2 C \sum_{i=0}^{n-1}\left|g^{i}(J)\right| \leq 2 C .
$$

Also by the mean value theorem there is a point $x_{n} \in J$ such that $\left|D g^{n}\left(x_{n}\right)\right|=$ $\left|g^{n}(J)\right| /|J|$. Therefore by (11) for every $y \in L$

$$
\left|D g^{n}(y)\right| \leq \exp (2 C)\left|D g^{n}\left(x_{n}\right)\right| \leq \exp (2 C) \frac{\left|g^{n}(J)\right|}{|J|} .
$$

Using the mean value theorem again and the fact that $(|L|-|J|) /|J|<\delta$, we see that

$$
\begin{aligned}
\left|g^{n}(L)\right| & \leq\left|g^{n}(J)\right|+\sup _{y \in L}\left|D g^{n}(y)\right|(|L|-|J|) \\
& \leq\left|g^{n}(J)\right|+\exp (2 C) \frac{\left|g^{n}(J)\right|}{|J|}(|L|-|J|) \\
& \leq(1+\delta \exp (2 C))\left|g^{n}(J)\right| \\
& \leq 2\left|g^{n}(J)\right| .
\end{aligned}
$$

This completes the induction step, so $\left|g^{n}(L)\right| \leq 2\left|g^{n}(J)\right|$ for all $n \geq 0$. The fact that $\sum_{i=0}^{\infty}\left|g^{i}(L)\right| \leq 2$ then follows from the fact that $\sum_{i=0}^{\infty}\left|g^{i}(J)\right| \leq 1$.

\subsection{Proof of Theorem 1.5.}

Theorem 1.5. Suppose $G<\operatorname{Diff}^{2}(\mathbf{R})$ is a non-abelian group with the property that every nontrivial element of $G$ has at most one fixed point. Then the function $\theta: \mathbf{R} \rightarrow \mathbf{R}$ defined by $\theta(x)=\nu(0, x)$ is a topological conjugacy from the action of $G$ on $\mathbf{R}$ to the standard action of $\operatorname{Aff}(\mathbf{R})$ on $\mathbf{R}$.

Proof. In Theorem 1.4 we showed that the function $\theta: \mathbf{R} \rightarrow \mathbf{R}$ defined by $\theta(x)=$ $\nu(0, x)$ is a surjective semi-conjugacy. It suffices to prove that $\theta$ is one-to-one. Note that if $\mu$ is the $G$-quasi-invariant measure provided by Theorem 3.1, then $\theta_{*}(\mu)$ is a Lebesgue measure on $\mathbf{R}$, the unique quasi-invariant measure for the standard action of $\operatorname{Aff}(\mathbf{R})$ on $\mathbf{R}$. The function $\theta$ is monotonic so $\theta^{-1}(y)$ consists of either a single point or a closed interval. If it is an interval $\left[x_{0}, x_{1}\right]$, then $\mu\left(\left[x_{0}, x_{1}\right]\right)=0$. The endpoints $x_{0}, x_{1}$ are in the support of $\mu$ but the interior $\left(x_{0}, x_{1}\right)$ is disjoint from the support of $\mu$.

We note that any interval $\left[x_{0}, x_{1}\right]=\theta^{-1}(y)$ must be wandering. That is, we must have $g\left(\left[x_{0}, x_{1}\right]\right) \cap\left[x_{0}, x_{1}\right]=\emptyset$ for all nontrivial $g \in G$. This is because if $g\left(\left[x_{0}, x_{1}\right]\right) \cap\left[x_{0}, x_{1}\right] \neq \emptyset$, the fact that $\theta$ is a semi-conjugacy implies that $g\left(\left[x_{0}, x_{1}\right]\right)=$ $\left[x_{0}, x_{1}\right]$ so $g$ would have two fixed points, $x_{0}$ and $x_{1}$.

Thus it suffices to show that the action of $G$ has no wandering intervals of the form $\theta^{-1}(y)$. Of course to do this we need only do it for a subgroup of $G$. In the proof of Theorem 1.3 we showed that the function $\phi: G \rightarrow \operatorname{Aff}(\mathbf{R})$ defined by $\phi(g)(x)=a x+b$, where $a=A(g)$ and $b=\nu(0, g(0))$, is an injective homomorphism. In particular if $A(h)=1$, then $\phi(h)(x)=x+\tau_{\mu}(h)$. Since we are assuming $G$ is not abelian, $A: G \rightarrow \mathbf{R}^{+}$is not trivial and neither is its kernel. Hence we will focus on a subgroup $G_{0}$ generated by two nontrivial elements, $g$ with $A(g)>1$, and $h$ with 
$A(h)=1$ and $\tau_{\mu}(h) \neq 0$. Replacing $h$ by $h^{-1}$ if necessary, we may assume $h(x)>x$ for all $x$.

Consider the quotient space of $\mathbf{R}$ by the action of $h$. There is a $C^{2}$ diffeomorphism from this quotient to $S^{1}$. We lift this diffeomorphism to a $C^{2}$ diffeomorphism of $\mathbf{R}$ and use it to conjugate our $G$ action to a $C^{2}$ action on $\mathbf{R}$ for which $h(y)=y+1$. We know $\phi(h)=x+c$ for some $c>0$ but composing $\phi$ with an inner automorphism of $\operatorname{Aff}(\mathbf{R})$ (i.e. an affine change of co-ordinates) we may assume $\phi(h)=x+1$ and $\phi(g)=\lambda x$ for some $\lambda>1$.

We first consider the case that $\lambda$ is irrational. Let $f=g h g^{-1}$. Since $h$ is in the kernel of $A$, a normal abelian subgroup, we have that $f$ commutes with $h$. We also have that $\phi(f)=x+\lambda$. Hence $f$ is the lift of a $C^{2}$ diffeomorphism of the circle $S^{1}$ with an irrational rotation number. By Denjoy's theorem this circle diffeomorphism has a dense orbit from which it follows that if $H$ is the group generated by $h$ and $f$, then the set $H(x)$ is dense in $\mathbf{R}$ for any $x$. Hence $H$ has no wandering intervals at all.

Thus we may assume $A(g)=p / q$ is rational. Note that $A(g)=p / q$ implies $g h^{q}=h^{p} g$ or $g(y+q)=g(y)+p$. Differentiating we see that for all $y \in \mathbf{R}$ we have $D g(y+q)=D g(y)$ and $D^{2} g(y+q)=D^{2} g(y)$. In particular $D g(y)$ and the derivative of $\log D g(y)$ are periodic and hence uniformly bounded.

We now want to show by contradiction that $G_{0}$ has no wandering intervals of the form $\theta^{-1}(y)$. Suppose $J=\left[x_{0}, x_{1}\right]=\theta^{-1}(y)$ is a wandering interval. Then for every nontrivial $g \in G_{0}, \phi(g)(y) \neq y$ because otherwise $g\left(\theta^{-1}(y)\right)=\theta^{-1}(y)$ and the endpoints of this $g$ invariant interval would be two fixed points for $g$.

We can conclude, in particular, that the elements $y_{n}=\phi(g)^{n}(y) \bmod (1)=$ $\phi\left(g^{n}\right)(y) \bmod (1)$ are all distinct, where $y \bmod (1)$ denotes $y$ minus the greateset integer in $y$. This is because otherwise $g(J)$ would equal $J$ for some $g \in G$. It follows that the intervals $J_{n}=\theta^{-1}\left(y_{n}\right)$ are all pairwise disjoint.

Since $\theta$ is a semiconjugacy and is $h$-equivariant, $\theta(x+n)=\theta(x)+n$ for all $n \in \mathbf{Z}$. As $\theta$ is monotonic and $\theta^{-1}(n)=n$ for all $n \in \mathbf{Z}$, we have $\theta^{-1}([n, n+1])=[n, n+1]$. In particular $\theta^{-1}([0,1])=[0,1]$, so since $J_{k}=\theta^{-1}\left(y_{k}\right)$ and $y_{k} \in[0,1]$ we have $J_{k} \subset[0,1]$ for all $k>0$. Recall that $\left|J_{k}\right|$ denotes the length of $J_{k}$ and observe that $J_{k}=g^{k}(J)-n_{k}$ for some integer $n_{k}$, so $\left|J_{k}\right|=\left|g^{k}(J)\right|$. Hence

$$
\sum_{k=0}^{\infty}\left|g^{k}(J)\right|=\sum_{k=0}^{\infty}\left|J_{k}\right| \leq 1,
$$

as the intervals $J_{k}$ are pairwise disjoint and all in [0,1].

Let $\delta>0$ be the value provided by Lemma 4.3 and choose an interval $L$ which contains $J$ in its interior and has length $|L|<(1+\delta)|J|$. Define $L^{\prime}=\theta(L)$ and note that it is a nontrivial closed interval with $y=\theta(J)$ in its interior. We may shrink $L^{\prime}$ slightly so that $\theta^{-1}$ of each endpoint is a single point (because $\theta^{-1}(x)$ is a nontrivial interval for at most countably many values of $x$ ). We redefine $L$ to be $\theta^{-1}$ of this shrunken $L^{\prime}$ and note that $L=\theta^{-1}\left(L^{\prime}\right)=\theta^{-1}(\theta(L))$. It follows that $g^{n}(L)=\theta^{-1}\left(\lambda^{n} L^{\prime}\right)$ for all $n>0$.

We observe that from Lemma 4.3 that

$$
\sum_{k=0}^{\infty}\left|g^{k}(L)\right| \leq 2
$$


However, $\theta\left(g^{k}(L)\right)=\lambda^{k} L^{\prime}$ and for $k$ sufficiently large $\lambda^{k}\left|L^{\prime}\right|>2$ which implies there is an interval $[m, m+1] \subset \lambda^{k} L^{\prime}$, with $m \in \mathbf{Z}$. This means that

$$
[m, m+1]=\theta^{-1}([m, m+1]) \subset \theta^{-1}\left(\lambda^{k} L^{\prime}\right)=g^{k}(L) .
$$

Hence $\left|g^{k}(L)\right| \geq 1$ for $k$ sufficiently large. This clearly contradicts equation (2) above. We conclude that the semi-conjugacy $\theta$ is one-to-one and hence a homeomorphism.

\section{REFERENCES}

[B] T. Barbot, Caracterisation des flots d'Anosov en dimension 3 par leurs feuilletages faibles, Ergodic Theory Dynam. Systems 15 (1995), no. 2, 247-270. MR 96d:58100

[dMvS] W. de Melo and S. van Strien One-dimensional Dynamics, Springer-Verlag, Berlin, (1993). MR 95a:58035

[FF1] B. Farb and J. Franks, Groups of homeomorphisms of one-manifolds, I: Nonlinear group actions, June 2001 preprint.

[FF2] B. Farb and J. Franks, Groups of homeomorphisms of one-manifolds, III: Nilpotent subgroups, Ergodic Theory and Dynam. Syst., to appear.

[FS] B. Farb and P. Shalen, Groups of real-analytic diffeomorphisms of the circle, Ergodic Theory and Dynam. Syst. 22 (2002), 835-844. MR 2003e:37030

[Gh] E. Ghys, Groups acting on the circle, IMCA, Lima, June 1999.

$[\mathrm{H}] \quad$ M. Hirsch, A stable analytic foliation with only exceptional minimal set, in Lecture Notes in Math., Vol. 468. Springer-Verlag, 1975. MR 52:13094

[Ho] O. Hölder, Die Axiome der Quantität und die Lehre vom Mass. Ber. Verh. Sachs. Ges. Wiss. Leipzig, Math. Phys. C1. 53, 1-64 (1901).

[K] N. Kovacevic, Möbius-like groups of homeomorphisms of the circle. Trans. Amer. Math. Soc. 351 (1999), no. 12, 4791-4822. MR 2000c:20074

[P] J. Plante, Solvable Groups acting on the line, Trans. Amer. Math. Soc. 278 (1983), 401-414. MR 85b:57048

[P2] J. Plante, Subgroups of continuous groups acting differentiably on the half-line, Ann. Inst. Fourier, Grenoble 341 (1984), 47-56. MR 86j:58020

[S] V. V. Solodov, Topological problems in the theory of dynamical systems. (Russian) Uspekhi Mat. Nauk 46 (1991), no. 4(280),93-114, 192 translation in Russian Math. Surveys 46 (1991), no. 4, 107-134. MR 92k:58203

Department of Mathematics, University of Chicago, 5734 University Ave., Chicago, ILLINOIS 60637

E-mail address: farb@math.uchicago.edu

Department of Mathematics, Northwestern University, Evanston, Illinois 60208

E-mail address: john@math.northwestern.edu 